\title{
Comprehensive Pharmacologic Management of Bipolar Depression
}

\author{
Alexander McGirr, MD, MSC ${ }^{1}$ \\ David J. Bond, MD, PhD 2,"
}

\author{
Address \\ ${ }^{1}$ Department of Psychiatry, University of British Columbia, 11th Floor, 2775 \\ Laurel Street, Vancouver, BC V5Z 1 M9, Canada \\ Email: alexander.mcgirr@alumni.ubc.ca \\ 2,* Department of Psychiatry, University of Minnesota, Room 516B - 717 Delaware \\ Street, Minneapolis, MN 55414, USA \\ Email: djbond@umn.edu
}

Published online: 18 May 2014

(C) Springer International Publishing AG 2014

Keywords Bipolar disorder - Depression - Pharmacotherapy - Lithium - Valproate - Lamotrigine - Olanzapine . Quetiapine - Aripiprazole - Ziprasidone - Risperidone - Lurasidone - Antidepressant - Modafinil - Armodafinil . Pramipexole · Fatty acids $\cdot \mathrm{N}$-acetylcysteine $\cdot$ Ketamine

\section{Opinion Statement}

Although mania is the defining feature of bipolar disorder (BD), depressive episodes are more frequent, at least as impairing, and come at high individual and societal costs. Historically, BD depression (BDD) has been under-studied, but in the last 10-15 years data from numerous placebo-controlled trials of mood stabilizers, second-generation antipsychotics, and other agents have allowed for the formulation of evidence-based treatment recommendations (Table 1). Abundant evidence exists for the efficacy of lithium, lamotrigine, quetiapine, and lurasidone in treating acute BDD. Lithium, lamotrigine, and quetiapine are also robustly effective in preventing depression during maintenance treatment, while data for lurasidone is lacking. 0ther second generation antipsychotics besides quetiapine are of limited benefit, with the possible exception of olanzapine, particularly in combination with fluoxetine, although weight gain and other metabolic abnormalities limit its use for many patients. Antidepressants remain controversial in the treatment of $\mathrm{BDD}$, and there is limited high-quality data to guide decision making. Nevertheless, the best available evidence suggests that SSRIs and bupropion, as adjuncts to mood stabilizers, are safe and effective in the treatment of acute BDD in patients with pure depression, no mixed symptoms, and no recent rapid cycling. However, they cannot be routinely recommended for maintenance therapy due to a lack of data regarding long-term efficacy and safety. Finally, a number of promising experimental agents with novel mechanisms of action deserve further study, including vigilance-promoting drugs such as modafinil and armodafinil; the dopamine agonist pramipexole; the glutathione donor $\mathrm{N}$-acetylcysteine; fatty acid supplementation; and the NMDA-receptor-antagonist ketamine. 


\section{Introduction}

Bipolar I disorder (BD) is characterized by episodes of mania and depression. It has a lifetime prevalence of approximately $1 \%$, meaning that over 3 million Americans are affected [1]. It is a highly disabling illness, with an early age of onset and a highly recurrent course, with patients typically spending half of their lives with mood symptoms $[2,3]$. The cost to society is correspondingly enormous, with direct and indirect illness-related expenses in the USA estimated at US $\$ 71.9$ billion annually [4].

Although manic and hypomanic episodes are the distinguishing features of $\mathrm{BD}$, the bulk of its symptom burden, disability, and health utilization costs results from its depressive phase. Longitudinal studies show that patients spend approximately three times as much time with depressive symptoms as with manic/hypomanic symptoms [3,5]. Depressive episodes take a major toll on relationships, productivity, and other areas of functioning, and are at least as disabling as mania $[6,7]$. Even mild depression has an unexpectedly large negative impact on functioning [8]. Other serious consequences of depression, including suicide, are more common in bipolar than unipolar depression [9]. From a health care systems perspective, de- pressed BD patients utilize outpatient and inpatient psychiatric services at a rate four to eight times greater than manic/hypomanic patients [10]. Thus, detecting and effectively treating bipolar depression (BDD) are essential skills for psychiatrists.

However, pharmacotherapy studies in BD have historically focused on mania, and BDD was relatively under-studied. Fortunately, that has recently changed, and in the last 10-15 years a broad array of mood stabilizers, second-generation antipsychotics, antidepressants, and other pharmacologic agents have been subjected to randomized controlled trials for BDD. Gaps in the literature undoubtedly remain. For example, there is still a vigorous debate regarding whether, and if so how, to appropriately use antidepressants for BDD. Furthermore, most of the clinical trials to date have been monotherapy studies in non-suicidal, non-psychotic patients, a situation not reflective of clinical practice. Finally, there is a dearth of studies directly comparing the relative efficacy of medications. Nonetheless, the current evidence base, supplemented by clinical experience, provides a framework to guide recommendations about the selection, sequencing and, for many patients, combination of the available treatments.

\section{Clinical assessment of BDD}

Assessment should focus on the onset, duration and severity of depressive symptoms; precipitating factors, including medication non-adherence, substance abuse, psychosocial stressors, and sleep patterns; and safety issues, including acute suicidality, homicidality, self-neglect, dependent-neglect, and psychosis. It is also critical to consider contextual factors, particularly the predominant polarity of mood episodes over the course of the illness, the presence of recent rapid cycling, and perhaps most importantly, the presence of mixed symptoms. As will be outlined below, these have important treatment implications, particularly with respect to the use of antidepressants.

\section{Treatment}

Lithium - the foundation of the evidence base for lithium in acute BDD consists of eight small placebo-controlled cross-over studies [11-18] (total $\mathrm{N}=135)$, and one imipramine-controlled trial $(\mathrm{N}=29)$ carried out in the 1960s-1970s. The mean response rate for lithium across studies was 
$75.8 \%$ (range $54 \%-100 \%$ ). A mean of $52 \%$ (38\%-78\%) of responders relapsed when blindly switched to placebo.

The only modern parallel-arm placebo-controlled trial of lithium for BDD was part of a large eight week study comparing quetiapine to placebo, in which a lithium arm was used as an internal validator [19]. Lithium was not superior to placebo in this study, $(62.5 \%$ vs. $55.8 \%$ clinical response; $62.5 \%$ vs. $55.0 \%$ remission).

Several clinically relevant points can be extracted from these studies. In the cross-over studies, lithium levels were generally in the upper end of the therapeutic range, between 1.0 and 1.2. In the negative parallel-arm study, the mean serum lithium level was 0.61, a level with ample data to suggest lower efficacy [20]. Coupled with an additional trial in which adding an antidepressant to lithium was superior to placebo only in patients with serum lithium level of $>0.8$ [21], this suggests that targeting the high end of the therapeutic range is most likely to lead to response. Another point relates to the required duration of treatment, with a duration of between 8 and 16 weeks typically required for response.

The efficacy of lithium in preventing depression during maintenance treatment has been robustly demonstrated in several 12 to 24 month placebo [22] or active comparator-controlled studies [14, 23-25]. These show that lithium is superior to placebo in preventing mania and depression, and equal to antidepressant in preventing depression without inducing mania. While two recent studies demonstrated that lithium was less effective than lamotrigine or quetiapine, these studies should be viewed with skepticism as they used enriched designs that favored the comparator [14, 25]. Finally, the anti-suicide effect of lithium has been well-established in numerous longterm studies and should not be discounted [26].

Taken together, this evidence creates a compelling case for using lithium in the acute and preventative treatment of BDD. Given the relatively long duration of treatment needed for response, it may not be appropriate, at least as monotherapy, for patients with severe depression requiring rapid response. Its potential for toxicity, and even lethality, in overdose may preclude its use in acutely suicidal outpatients. Nonetheless, its robust maintenance efficacy and long-term anti-suicide properties mandate its consideration for most BD patients.

Valproate - has not been well-studied in BDD, and perhaps for this reason is seldom thought of as a treatment option. Nonetheless, its efficacy has been examined in four small six to eight week placebo-controlled trials (total $\mathrm{N}=142$ ), recently subjected to meta-analysis [27•]. The pooled analysis suggested a significant benefit for valproate, with $40.6 \%$ of valproate-treated vs. $24.3 \%$ of placebo-treated patients meeting criteria for clinical remission. Valproate was well tolerated, with comparable drop-out rates in medication-treated and placebo-treated patients.

There is similarly limited information on valproate prophylaxis in preventing BDD. Data from the BALANCE trial, a randomized open-label maintenance study $(\mathrm{N}=330)$, demonstrated significantly superior prevention of depression with lithium over valproate [28•]. Other maintenance RCTs suggest benefit of valproate over placebo, and possibly lithium [29, 30]. In the challenging rapid-cycling population, a small head-to-head study of valproate and lithium suggested equipoise [31]. Clearly, the efficacy of 
valproate in treating and preventing $\mathrm{BDD}$ remains a matter of some conjecture.

Lamotrigine- has been studied in five 8-10 week placebo-controlled monotherapy trials in BDD (total $\mathrm{N}=1072$ ) [32•,33], and a placebo-controlled add-on study to lithium [34]. A meta-analysis of the monotherapy studies suggested that lamotrigine was significantly, but modestly, more effective than placebo, with a very small effect size (0.12) and a NNT of 13. It was more robustly effective in patients with severe depression, due to lower placebo response rates. The add-on study reported that adjunctive lamotrigine was significantly superior to placebo (51.6\% lamotrigine response vs. $31.7 \%$ placebo). However a limitation is that this finding was contingent on classifying the emergence of hypo/manic episodes as response. Lamotrigine was well tolerated in these studies, with a very low incidence of rash and few other side effects.

The relatively disappointing results of these RCTs fly in the face of many clinicians' positive experience with lamotrigine. While it has sometimes been claimed that the lamotrigine studies failed due to unusually high placebo response rates, these were in fact similar to those in five large quetiapine studies (reviewed below). Nonetheless, several shortcomings of these trials deserve mentioning. The first is the low, likely subtherapeutic final dose of lamotrigine $(200 \mathrm{mg}$ in three monotherapy studies, $50 \mathrm{mg}$ or $200 \mathrm{mg}$ in one study, and a flexible dose of 100-400 $\mathrm{mg}$ in the final study). In clinical settings, lamotrigine is often prescribed at significantly higher doses, not uncommonly up to $500 \mathrm{mg}$ [35]. The second is the long titration, required to minimize the risk of rash, leaving patients only between two and three weeks at the target dose before study end. This is in stark contrast with other agents used in BDD, in which the target dose was typically reached in the initial days of the study. Thus, the trials unfortunately do not reflect the efficacy of optimally-prescribed lamotrigine.

In contrast to its limited efficacy in acute-phase clinical trials, lamotrigine was consistently superior to placebo in maintenance studies. Two 18-month placebo-controlled trials in BDI patients showed lamotrigine was significantly better than placebo in preventing depression [36, 37], with a more modest, but still significant anti-manic effect [14]. Although lamotrigine was superior to lithium in preventing depression, this finding is questionable given the enriched study design favoring lamotrigine.

Considering both the clinical trial data and clinical experience, lamotrigine is an effective and well tolerated treatment for BDD. Like lithium, it may be best utilized in patients with non-severe depression who are not acutely suicidal. It is well suited to patients with a highly recurrent depressive illness course, and those with tolerability issues to other medications.

\section{Second-generation antipsychotics (SGAs)}

Although first-generation antipsychotics (FGAs) are highly effective in treating and preventing mania, they actually increase depressive symptoms and episodes $[38,39]$. In contrast, SGAs have greater affinity for 5HT-2 than D2 receptors, providing a theoretical mechanism for antidepressant properties [40]. This, coupled with the clinical trial finding that SGAs decreased depressive symptoms in BD patients with mixed episodes, stimulated research examining SGAs in BDD. 
Olanzapine (OLZ) - was the first SGA studied in acute BDD in a large $(\mathrm{N}=833)$ RCT comparing OLZ to placebo and a small olanzapine-fluoxetine combination (OFC) arm $(\mathrm{n}=86)$ [41]. OLZ was statistically superior to placebo (remission $32.8 \%$ vs. $24.5 \%$; response $39.0 \%$ vs. $30.4 \%$ ), yet it was outperformed by OFC ( $48.8 \%$ remission; $56.1 \%$ response). The effect sizes observed in this study were small for OLZ (0.32) and moderate for OFC (0.68). Furthermore, OLZ mostly led to improvement in sleep and appetite (known side effects of the medication), while OFC was superior in treating core depressive symptoms. These results were recently confirmed in a replication study with OLZ [42] and a seven week RCT comparing OFC to lamotrigine [43]. A meta-analysis of OFC trials demonstrated superiority to both olanzapine monotherapy and placebo, but equipoise with lamotrigine [44]. As expected, significant rates of weight gain and metabolic abnormalities were reported with OLZ and OFC. Given its small effect in BDD, coupled with significant tolerability concerns, we argue that OLZ has a limited role in the clinical management of BDD.

Quetiapine-and its extended release formulation have the largest clinical trial database of any treatments in BDD. This includes five placebo-controlled studies with a total $\mathrm{N}$ of approximately 3,000 patients [19, 45, 46, $47 \bullet, 48]$. These studies consistently found quetiapine $300 \mathrm{mg}$ or $600 \mathrm{mg}$ to be superior to placebo in treating core depressive symptoms in BDD, with a rapid onset of action, separating from placebo typically after one week. Furthermore, quetiapine was similarly effective in rapid-cycling patients, and was relatively well-tolerated, with sedation and, less commonly, weight gain being the limiting concerns. In two of the studies, quetiapine was superior to lithium and paroxetine monotherapy, respectively; however we encourage healthy skepticism as these comparators were under-dosed.

Quetiapine, alone or in combination with mood stabilizers, is also more effective than placebo in preventing depression during maintenance treatment [25, 49]. As noted above, in one maintenance study, quetiapine was superior to lithium in preventing depression, but employed an enriched design favoring quetiapine [25].

One clinically relevant question that is not answered by these studies is whether lower doses than 300-600 mg may be effective. This question is particularly germane given that doses of 50-150 mg were found to be effective in treating unipolar depression [50]. Our clinical experience suggests that starting with $50 \mathrm{mg}$ HS and titrating to 150-200 mg over seven to ten days is effective and well-tolerated for most patients. If daytime sedation is problematic, then using the extended release formulation at suppertime may ameliorate this problem. Quetiapine's robust effectiveness, which is supported by our clinical experience, its relatively rapid onset of action, and its clear effica$\mathrm{cy}$ in ring maintenance treatment mean that quetiapine may be considered for a broad range of BDD patients.

Aripiprazole and Ziprasidone-the effectiveness of essentially all SGAs in treating mania [51] engendered the hope that a similar class effect may exist for $\mathrm{BDD}$. Unfortunately, this is not the case, as evidenced by negative studies of both aripiprazole and ziprasidone. Aripiprazole was not significantly better than placebo at study endpoint in two eight-week RCTs (total N=749) [52]; and also did not separate from placebo in a smaller adjunctive study in which it was added to mood stabilizer+citalopram [53]. Some have criticized these studies for the high starting dose $(10 \mathrm{mg})$ and mean study dose (15-18 $\mathrm{mg}$ ) of aripiprazole, particularly as 
doses in the 2-5 mg range are effective as augmentation to SSRIs in unipolar depression, and since extrapyramidal symptoms are frequently problematic with high starting doses. Indeed, the drop-out rates in aripiprazole-treated patients exceeded those of placebo-treated patients in both studies. Nonetheless, unlike lamotrigine or quetiapine, aripiprazole was not more effective than placebo in preventing depression during maintenance treatment either as monotherapy or in combination with other medications [54-57], further arguing against efficacy. Similarly, ziprasidone did not separate from placebo in two six-week RCTs (total $\mathrm{N}=885$ ) [58・]. Interestingly, it was superior to placebo (clinical response $52.9 \%$ vs. $28.9 \%$ ) in a small study of bipolar II and unipolar patients with depression and minimal hypomanic symptoms [59]. In any case, neither of these medications can currently be recommended in treating BDD.

Risperidone - has not been studied in placebo controlled-trials in BDD. The available evidence, however, suggests a lack of efficacy. In a randomized open-label study $(\mathrm{N}=66)$ comparing it, lamotrigine, and inositol in treatment-resistant BDD [60], risperidone had the lowest response rate of the three treatments ( $4.6 \%$, vs. $23.8 \%$ for lamotrigine and $17.4 \%$ for inositol). Furthermore, risperidone long-acting injectable formulation was not better than placebo in preventing depression in a 52-week maintenance study [61].

Lurasidone- is the most recently studied SGA in BDD. Monotherapy and adjunctive therapy (to mood stabilizers) were both more effective than placebo in treating BDD in two six-week RCTs (total $\mathrm{N}=853$ )

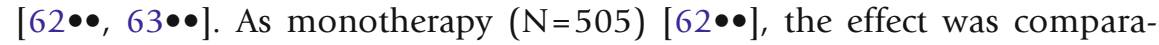
ble to that observed for quetiapine. A $20-40 \mathrm{mg}$ dose appeared to be equally effective to $60-120 \mathrm{mg}$, and was better tolerated. Post-hoc analyses showed that lurasidone was equally effective in patients with pure and mixed depression, a noteworthy finding given the paucity of treatments for mixed BDD. It was well-tolerated, and was almost weight-neutral (mean difference $0.0-0.6 \mathrm{~kg} / 6$ weeks monotherapy; $0.2 \mathrm{~kg} / 6$ weeks adjunctive). Akathisia was more common than in placebo-treated patients (7.8-10.9 \% vs. $2.4 \%$ monotherapy; $7.7 \%$ vs. $4.3 \%$ adjunctive) as were extra-pyramidal symptoms (4.9-9.0 \% vs. $2.4 \%$ monotherapy; $15.3 \%$ vs. $9.8 \%$ adjunctive). As it is a newer agent, our clinical experience with lurasidone is relatively limited. Nonetheless, our initial impression is that lurasidone 20-40 mg with meals (required for optimal absorption) can be effective for many patients, and might be particularly considered in those for whom weight gain is a concern.

\section{Antidepressants}

At least half, and up to $70 \%$, of $\mathrm{BD}$ patients are prescribed antidepressants (ADs), the majority for at least one year [64, 65]. Despite this, ADs remain the most poorly studied and most controversial medications in the treatment of BDD, and questions persist about their efficacy and safety, particularly their purported propensity to induce manic and hypomanic episodes.

These concerns are partly a historical consequence of the early $\mathrm{AD}$ studies for $\mathrm{BD}$. These trials were primarily long-term prophylaxis studies examining the tricyclic $\mathrm{AD}$ imipramine, most commonly prescribed as monotherapy. They clearly demonstrated that imipramine-treated patients were more likely than placebo-treated patients to experience a manic relapse [23, 24]. Although 


\section{Table 1. Treatment recommendations}

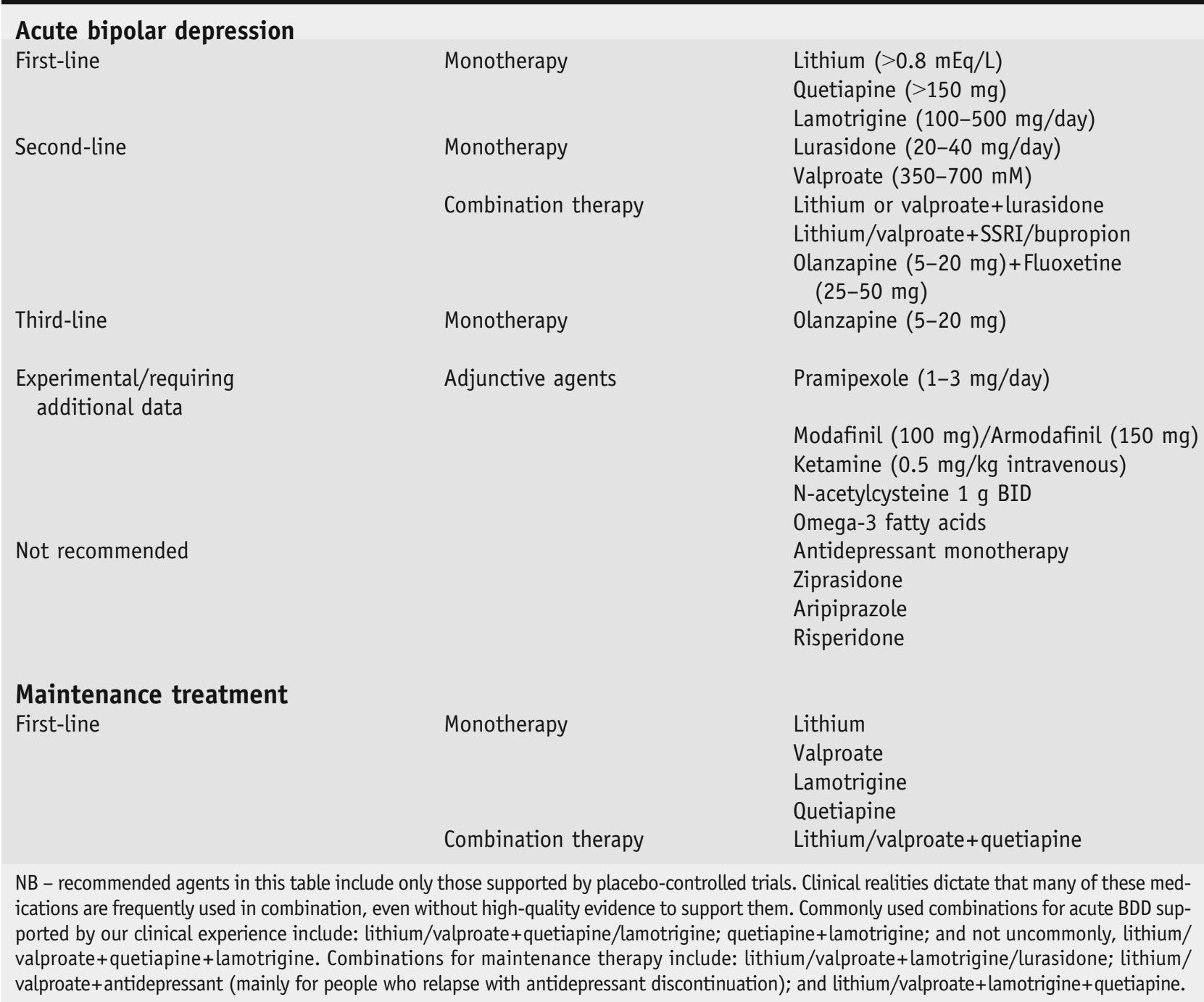

combining imipramine with lithium appeared to reduce the risk of mania, the combination was no more effective than lithium monotherapy in preventing overall relapse (i.e., into either mania or depression). Largely as a result of these studies, a firm belief took hold that ADs had limited effectiveness in BDD, and were associated with a worsening of the manic pole of the illness.

This view persisted despite a revolution in $\mathrm{AD}$ treatment, with the introduction of the SSRIs and other classes of modern antidepressants. Thus, it came as a surprise when OFC was shown to be superior to both placebo and OLZ in acute BDD, without increasing the risk of manic switch (as reviewed above) [41]. Furthermore, a meta-analysis (including the OFC trial, and several other small studies) demonstrated that patients prescribed a modern $\mathrm{AD}$ as an adjunct to an antimanic medication were approximately twice as likely as those who received adjunctive placebo to respond to short-term treatment, and no more likely to experience a manic relapse 
Table 2. International Society for Bipolar Disorders recommendations on antidepressants in bipolar depression (adapted from Pacchiarotti et al., 2013)

\begin{tabular}{|c|c|}
\hline $\begin{array}{l}\text { Clinical } \\
\text { circumstances }\end{array}$ & Recommendation \\
\hline \multirow[t]{2}{*}{ Initiation } & $\begin{array}{l}\text { - ADs should generally not be considered first-line treatments, but rather should be reserved } \\
\text { for people who have failed first-line medications (lithium, quetiapine, or lamotrigine) }\end{array}$ \\
\hline & $\begin{array}{l}\text { - They can be considered first-line if there is a known history of response and lack of } \\
\text { AD-induced mania }\end{array}$ \\
\hline $\begin{array}{l}\text { Mixed states and rapid } \\
\text { cycling }\end{array}$ & $\begin{array}{l}\text { - ADs should be prescribed only for pure BDD, avoided in people with mixed symptoms, recent } \\
\text { rapid cycling, or a past history of AD-induced mania, and should be discontinued if these } \\
\text { conditions develop }\end{array}$ \\
\hline $\begin{array}{l}\text { Concurrent } \\
\text { medications }\end{array}$ & - ADs should only be prescribed as adjuncts to anti-manic agents \\
\hline Selection & $\begin{array}{l}\text { - Preference should be given to ADs with a low propensity for manic switch, such as SSRIs } \\
\text { and bupropion }\end{array}$ \\
\hline Duration of treatment & $\begin{array}{l}\text { - TCAs and SNRIs should generally be avoided } \\
\text { - As there are no placebo-controlled maintenance data, antidepressants should be discontinued } \\
\text { after a reasonable period of wellness ( } 2-4 \text { months). In patients who clearly relapse with } \\
\text { disconsolation, reinstitution of the AD for maintenance treatment can be considered. }\end{array}$ \\
\hline
\end{tabular}

[66]. The same meta-analysis found that TCAs were significantly more likely to lead to manic switch than other ADs, suggesting that extension of TCA-associated fears were largely unfounded.

Unfortunately, the controversy regarding AD use continues, as the only other large trial of modern ADs besides the OFC study demonstrated no benefit over placebo with the addition of paroxetine or bupropion to a mood stabilizer for six months of treatment [67]. The most current summary of the risks and benefits associated with ADs in BDD comes from a recent meta-analysis of shortterm studies, showing that modern ADs as adjuncts to mood stabilizers are modestly more effective than placebo in the short-term treatment of BDD $(p=0.06)[68 \bullet \bullet]$, though with a small effect size. Nonetheless, they were no more likely than placebo to lead to manic or hypomanic switch.

Despite the presence of meta-analytic data for ADs, numerous questions remain regarding their suitability in the treatment of BDD. Foremost is the possibility of differences in efficacy between ADs. The premise for unequal efficacy is not without basis, given data from meta-analyses suggesting differences in MDD [69]. This is highly relevant to $\mathrm{BDD}$, as only four ADs (imipramine, paroxetine, fluoxetine, and bupropion) have been compared to placebo for BDD, and two of these (imipramine and paroxetine) are not commonly used in clinical practice. It is thus unwarranted and premature to generalize the results of the above meta-analysis to any $\mathrm{AD}$ beyond these four medications. The situation is even worse with respect to maintenance data- not a single placebo-controlled study has assessed the maintenance efficacy of any modern AD for BDD. Randomized open-label data [70] and case series [71] suggest benefits to $\mathrm{AD}$ continuation, but definitive data are lacking.

There is similar uncertainty with respect to differences in safety. As discussed, meta-analyses suggest that TCAs are more likely to lead to manic 
switches than other ADs [66]. Whether there are switch differences between modern $\mathrm{AD}$ classes is less clear, though some evidence suggests that venlafaxine (which has similar effects on neurotransmitters as TCAs) is associated with higher switch rates than SSRIs or bupropion $[72,73]$.

Finally, whether $\mathrm{AD}$ use should be limited to some sub-populations within BDD is also unclear. Some data suggest that ADs are less likely to cause affective switches in BDII than BDI [74], and patients with rapid cycling appear to do more poorly with $\mathrm{AD}$ treatment than patients without [70]. It has also been recommended that if ADs are to be used, that they be prescribed only for patients with pure BD depression and be avoided, and in fact discontinued, in patients with mixed symptoms [75].

In the absence of high-quality data for ADs, an expert panel recently provided recommendations, which are summarized in Table 2 [75].

\section{Experimental treatments}

\section{Modafinil and armodafinil}

Spurred on by data suggesting efficacy for vigilance-promoting medications as augmentation agents to SSRIs in MDD [76], several RCTs have been performed for BDD. One moderately-sized six-week placebo-controlled trial $(\mathrm{N}=85)$ demonstrated efficacy for adjunctive modafinil $100-200 \mathrm{mg} /$ day, with $39 \%$ of modafinil-treated patients remitting compared to $18 \%$ of placebo-treated patients [77]. Two additional eight-week studies $(\mathrm{N}=257$ and $\mathrm{N}=393)$ randomized $\mathrm{BDD}$ patients to receive adjunctive armodafinil or placebo [78•,79]. They produced equivocal results, with the first study reporting a statistical trend for superiority of armodafinil, and the second a small effect size (0.28) that only emerged by week six. Modafinil and armodafinil were well tolerated, with particularly low rates of hypomanic/manic switch $(4.8 \%$ modafinil vs. $11.3 \%$ placebo) [77], and $2.3 \%$ armodafinil vs. $5.4 \%$ placebo [78•]. Taken together, these data suggest that vigilance-promoting agents are associated with modest clinical benefit, yet their safety and tolerability may suggest a role, particularly where fatigue and concentration difficulties lead to impairment.

\section{Pramipexole}

Two small trials have assessed pramipexole, a D2/D3 agonist, for BDD. In the first, 22 treatment-refractory, predominantly $\mathrm{BD} 1$ patients were randomized to six weeks of adjunctive pramipexole $(1-2.5 \mathrm{mg} / \mathrm{day})$ or placebo [80]. Sixty-seven percent of pramipexole treated patients met criteria for clinical response, compared to $20 \%$ of placebo treated patients. This was followed by a small six-week placebo-controlled trial of pramipexole $1-3 \mathrm{mg} /$ day in $21 \mathrm{BDII}$ patients. Pramipexole separated from placebo beginning at week three, and by study endpoint the pramipexole group had higher rates of response (60\% vs. $9 \%$ ) and remission (40\% vs. $9 \%$ ) [81]. Manic switch rates were low (2/22 pramipexole-treated and 2/21 placebo-treated). Based on these trials, 
pramipexole deserves further attention for BDD, but the small evidence base to date limits its use in routine clinical practice.

\section{Fatty Acids}

There is growing interest in fatty acid supplementation for BDD, stimulated by indications of efficacy in MDD [82]. A small $(\mathrm{N}=30) 16$-week placebocontrolled trial of adjunctive eicosapentanoic acid (EPA) $3.080 \mathrm{~g} /$ day and docosahexanoic acid (DHA) $1.680 \mathrm{~g}$ twice daily in BDI and BDII patients reported improvement in depressive symptoms compared to placebo [83]. A subsequent 12-week trial in 75 moderately depressed BDI and BDII patients with or without concomitant medications [84] randomized them to $1 \mathrm{~g}$ or $2 \mathrm{~g}$ daily of EPA and demonstrated similar benefit, with a modest decrease in depressive symptoms relative to placebo (effect size 0.34). However, these positive results were not replicated in a moderately-sized $(\mathrm{N}=121) 12$-week placebo-controlled trial of $6 \mathrm{~g} /$ day EPA in rapid-cycling patients [85], or a small $(\mathrm{N}=45)$ placebo controlled RCT using $3 \mathrm{~g} /$ day of EPA and $2 \mathrm{~g} /$ day of DHA [86]. Common side-effects of fatty acid supplementation included loose stools, gastrointestinal upset, and unpleasant aftertaste.

Currently, the small evidence base is not sufficient to support robust efficacy of fatty acid supplementation in BDD. Definitive recommendations, including the optimal dosing regimen, will require high-quality RCTs.

\section{N-Acetyl Cysteine}

Based on data suggesting increased inflammation and oxidative stress in BD, the glutathione donor N-acetylcysteine (NAC) has been studied for BDD. In an eight-week open-label trial, $149 \mathrm{BDI}$ and BDII patients with moderate depression showed significant improvement in depressive symptoms with adjunctive NAC $1 \mathrm{~g}$ BID [87]. However, when these same patients were randomized to continue NAC or switched to placebo for 24 weeks [88], similar proportions experienced a depressive recurrence. Based on these results, there is currently insufficient data to recommend adjunctive NAC in either acute BDD or in maintenance treatment.

\section{Ketamine}

The NMDA antagonist ketamine has garnered significant attention as a promising agent in the rapid treatment of depressive episodes. For BDD, two small placebo-controlled crossover trials (total $\mathrm{N}=34$ ) have assessed the efficacy of a single dose of intravenous ketamine $(0.5 \mathrm{mg} / \mathrm{kg})[89,90]$. Both studies produced positive results, with $43 \%$ and $44 \%$, respectively, of ketamine-treated patients responding after one day, while no placebotreated patient met response criteria. The effect waned over the one to two week follow-up period. In line with the challenges inherent to treating $\mathrm{BDD}$, a recently performed meta-analysis [91] demonstrated lower clinical effect in BDD samples than MDD samples (0.68 vs. 1.07). Based on these preliminary data, ketamine may prove to be a useful agent, particularly in severely depressed patients needing a rapid response. However, larger parallel-design trials, ideally with multiple ketamine infusions, a longer follow-up 


\section{Conclusion}

period, and an adequate placebo condition that mimics ketamine's psychotomimetic side effects are required to determine the role of ketamine, as well as its target population.

There have been numerous developments and high quality randomized controlled evidence in the treatment of BDD in the last two decades. The evidence base now supports several efficacious and well tolerated first-line agents, as well as appropriate combination strategies. The field is poised to make new advances, with numerous experimental pharmacological agents with novel mechanisms of action emerging. Yet, we believe that several fundamental and longstanding issues are deserving of immediate attention from researchers and clinicians alike, notably the appropriate use of antidepressants in BDD.

\section{Compliance with Ethics Guidelines}

\section{Conflict of Interest}

Alexander McGirr declares that he has no conflicts of interest.

David J. Bond reports personal fees and non-financial support from Sunovion; Advisory board and/or honoraria for lecture from Lundbeck, Janssen-Ortho and Otsuka; grants and personal fees from Pfizer; personal fees and non-financial support for advisory board positions, travel and honorarium for lecture from Astra-Zeneca and BMS, outside of the submitted work.

\section{Human and Animal Rights and Informed Consent}

This article does not contain any studies with human or animal subjects performed by any of the authors.

\section{References and Recommended Reading}

Papers of particular interest, published recently, have been highlighted as:

- Of importance

$\bullet \quad$ Of major importance

1. Merikangas KR et al. Lifetime and 12-month preva-

4. Dilsaver SC. An estimate of the minimum economic lence of bipolar spectrum disorder in the National Comorbidity Survey replication. Arch Gen Psychiatry. 2007;64(5):543-52.

2. Perlis RH et al. Long-term implications of early onset in bipolar disorder: data from the first 1000 participants in the systematic treatment enhancement program for bipolar disorder (STEP-BD). Biol Psychiatry. 2004;55(9):875-81.

3. Judd LL et al. Psychosocial disability and work role function compared across the long-term course of bipolar I, bipolar II and unipolar major depressive disorders. J Affect Disord. 2008;108(12):49-58. burden of bipolar I and II disorders in the United States: 2009. J Affect Disord. 2011;129(1-3):79-83.

5. Kupka RW et al. Three times more days depressed than manic or hypomanic in both bipolar I and bipolar II disorder. Bipolar Disord. 2007;9(5):531-5.

6. Calabrese JR et al. Impact of depressive symptoms compared with manic symptoms in bipolar disorder: results of a U.S. community-based sample. J Clin Psychiatry. 2004;65(11):1499-504.

7. Simon GE et al. Mood symptoms, functional impairment, and disability in people with bipolar disorder: specific effects of mania and depression. J Clin Psychiatry. 2007;68(8):1237-45. 
8. Altshuler LL et al. Subsyndromal depressive symptoms are associated with functional impairment in patients with bipolar disorder: results of a large, multisite study. J Clin Psychiatry. 2006;67(10):1551-60.

9. Rihmer Z, Kiss K. Bipolar disorders and suicidal behaviour. Bipolar Disord. 2002;4 Suppl 1:21-5.

10. Fu AZ et al. The economic burden of bipolar-related phases of depression versus mania. Drug Benefit Trends. 2004;16:569-75.

11. Baron $\mathrm{M}$ et al. Lithium carbonate response in depression. Prediction by unipolar/bipolar illness, average-evoked response, catechol-O-methyl transferase, and family history. Arch Gen Psychiatry. 1975;32(9):1107-11.

12. Donnelly EF et al. Prediction of antidepressant responses to lithium. Am J Psychiatry.

1978;135(5):552-6.

13. Goodwin FK et al. Lithium response in unipolar versus bipolar depression. Am J Psychiatry. 1972;129(1):44-7.

14. Goodwin GM et al. A pooled analysis of 2 placebocontrolled 18-month trials of lamotrigine and lithium maintenance in bipolar I disorder. J Clin Psychiatry. 2004;65(3):432-41.

15. Mendels J. Lithium in the treatment of depression. Am J Psychiatry. 1976;133(4):373-8.

16. Noyes Jr R et al. Lithium treatment of depression. Compr Psychiatry. 1974;15(3):187-93.

17. Stokes PE et al. Efficacy of lithium as acute treatment of manic-depressive illness. Lancet. 1971;1(7713):1319-25.

18. Greenspan $\mathrm{K}$ et al. Catecholamine metabolism in affective disorders. 3. MHPG and other catecholamine metabolites in patients treated with lithium carbonate. J Psychiatr Res.

1970;7(3):171-83.

19. Young $\mathrm{AH}$ et al. A double-blind, placebo-controlled study of quetiapine and lithium monotherapy in adults in the acute phase of bipolar depression (EMBOLDEN I). J Clin Psychiatry. 2010;71(2):15062.

20. Nolen WA, Weisler RH. The association of the effect of lithium in the maintenance treatment of bipolar disorder with lithium plasma levels: a post hoc analysis of a double-blind study comparing switching to lithium or placebo in patients who responded to quetiapine (Trial 144). Bipolar Disord. 2013;15(1):100-9.

21. Nemeroff CB et al. Double-blind, placebo-controlled comparison of imipramine and paroxetine in the treatment of bipolar depression. Am J Psychiatry. 2001;158(6):906-12.

22. Fieve RR, Kumbaraci T, Dunner DL. Lithium prophylaxis of depression in bipolar I, bipolar II, and unipolar patients. Am J Psychiatry. 1976;133(8):925-9.
23. Prien RF, Klett CJ, Caffey Jr EM. Lithium carbonate and imipramine in prevention of affective episodes. A comparison in recurrent affective illness. Arch Gen Psychiatry. 1973;29(3):420-5.

24. Prien RF et al. Drug therapy in the prevention of recurrences in unipolar and bipolar affective disorders. Report of the NIMH Collaborative Study Group comparing lithium carbonate, imipramine, and a lithium carbonate-imipramine combination. Arch Gen Psychiatry. 1984;41(11):1096-104.

25. Weisler RH et al. Continuation of quetiapine versus switching to placebo or lithium for maintenance treatment of bipolar I disorder (Trial 144: a randomized controlled study). J Clin Psychiatry. 2011;72(11):1452-64.

26. Cipriani A et al. Lithium in the prevention of suicidal behavior and all-cause mortality in patients with mood disorders: a systematic review of randomized trials. Am J Psychiatry. 2005;162(10):1805-19.

27. Bond DJ, Lam RW, Yatham LN. Divalproex sodium versus placebo in the treatment of acute bipolar depression: a systematic review and meta-analysis. J Affect Disord. 2010;124(3):228-34.

A meta-analysis with five small placebo-controlled 6-8 week RCTs representing $(\mathrm{N}=142)$ demonstrating that valproate is effective in the treatment of acute BD-D (39.3\% vs $17.5 \%$ response). An important finding requiring replication with high-quality, well conducted placebo-controlled RCT trials

28. Geddes JR et al. Lithium plus valproate combination therapy versus monotherapy for relapse prevention in bipolar I disorder (BALANCE): a randomised open-label trial. Lancet. 2010;375(9712):385-95.

Large open-label RCT of lithium $(\mathrm{N}=330)$ and valproate monotherapy compared to lithium and valproate combination, demonstrating a significant benefit for lithium and lithium combination therapy in the prevention of depressive episodes

29. Bowden CL et al. A randomized, placebo-controlled 12-month trial of divalproex and lithium in treatment of outpatients with bipolar I disorder. Divalproex Maintenance Study Group. Arch Gen Psychiatry. 2000;57(5):481-9.

30. Gyulai L et al. Maintenance efficacy of divalproex in the prevention of bipolar depression.

Neuropsychopharmacology. 2003;28(7):1374-82.

31. Calabrese JR et al. A 20-month, double-blind, maintenance trial of lithium versus divalproex in rapid-cycling bipolar disorder. Am J Psychiatry. 2005;162(11):2152-61.

32. Geddes JR, Calabrese JR, Goodwin GM. Lamotrigine for treatment of bipolar depression: independent meta-analysis and meta-regression of individual patient data from five randomised trials. Br J Psychiatry. 2009;194(1):4-9.

A meta-analysis of five trials of lamotrigine in the treatment of acute BD-D ( $\mathrm{N}=1072)$. The pooled analyses demonstrate that lamotrigine is an efficacious treatment in BD-D, however it is associated with a small effect size and high NNT 
33. Calabrese JR et al. Lamotrigine in the acute treatment of bipolar depression: results of five double-blind, placebo-controlled clinical trials. Bipolar Disord. 2008;10(2):323-33.

34. van der Loos ML et al. Efficacy and safety of lamotrigine as add-on treatment to lithium in bipolar depression: a multicenter, double-blind, placebocontrolled trial. J Clin Psychiatry. 2009;70(2):22331.

35. Calabrese JR et al. A double-blind, placebo-controlled, prophylaxis study of lamotrigine in rapidcycling bipolar disorder. Lamictal 614 Study Group. J Clin Psychiatry. 2000;61(11):841-50.

36. Bowden CL et al. A placebo-controlled 18-month trial of lamotrigine and lithium maintenance treatment in recently manic or hypomanic patients with bipolar I disorder. Arch Gen Psychiatry. 2003;60(4):392-400.

37. Calabrese JR et al. A placebo-controlled 18-month trial of lamotrigine and lithium maintenance treatment in recently depressed patients with bipolar I disorder. J Clin Psychiatry. 2003;64(9):1013-24.

38. Zarate Jr CA, Tohen M. Double-blind comparison of the continued use of antipsychotic treatment versus its discontinuation in remitted manic patients. Am J Psychiatry. 2004;161(1):169-71.

39. Bond DJ, Pratoomsri W, Yatham LN. Depot antipsychotic medications in bipolar disorder: a review of the literature. Acta Psychiatr Scand Suppl. 2007;434:3-16.

40. Tollefson GD et al. Depressive signs and symptoms in schizophrenia: a prospective blinded trial of olanzapine and haloperidol. Arch Gen Psychiatry. 1998;55(3):250-8.

41. Tohen $\mathrm{M}$ et al. Efficacy of olanzapine and olanzapine-fluoxetine combination in the treatment of bipolar I depression. Arch Gen Psychiatry. 2003;60(11):1079-88.

42. Tohen $\mathrm{M}$ et al. Randomised, double-blind, placebocontrolled study of olanzapine in patients with bipolar I depression. Br J Psychiatry. 2012;201(5):37682.

43. Brown EB et al. A 7-week, randomized, double-blind trial of olanzapine/fluoxetine combination versus lamotrigine in the treatment of bipolar I depression. J Clin Psychiatry. 2006;67(7):1025-33.

44. Silva MT et al. Olanzapine plus fluoxetine for bipolar disorder: a systematic review and meta-analysis. J Affect Disord. 2013;146(3):310-8.

45. Calabrese JR et al. A randomized, double-blind, placebo-controlled trial of quetiapine in the treatment of bipolar I or II depression. Am J Psychiatry. 2005;162(7):1351-60.

46. Thase ME et al. Efficacy of quetiapine monotherapy in bipolar I and II depression: a double-blind, placebo-controlled study (the BOLDER II study). J Clin Psychopharmacol. 2006;26(6):600-9.
47. Suppes T et al. Effectiveness of the extended release formulation of quetiapine as monotherapy for the treatment of acute bipolar depression. J Affect Disord. 2010;121(1-2):106-15.

A well sized 8-week placebo controlled RCT $(\mathrm{N}=280)$ of quetiapine extended release $(300 \mathrm{mg} / \mathrm{d})$ demonstrating efficacy in acute BD-D (54.1\% vs $39.4 \%$ remission)

48. McElroy SL et al. A double-blind, placebo-controlled study of quetiapine and paroxetine as monotherapy in adults with bipolar depression (EMBOLDEN II). J Clin Psychiatry. 2010;71(2):163-74.

49. Suppes T et al. Maintenance treatment for patients with bipolar I disorder: results from a north american study of quetiapine in combination with lithium or divalproex (trial 127). Am J Psychiatry.

2009;166(4):476-88.

50. Thase ME et al. Quetiapine XR monotherapy in major depressive disorder: a pooled analysis to assess the influence of baseline severity on efficacy. Int Clin Psychopharmacol. 2013;28(3):113-20.

51. Cipriani A et al. Comparative efficacy and acceptability of antimanic drugs in acute mania: a multipletreatments meta-analysis. Lancet. 2011;378(9799):1306-15.

52. Thase ME et al. Aripiprazole monotherapy in nonpsychotic bipolar I depression: results of 2 randomized, placebo-controlled studies. J Clin Psychopharmacol. 2008;28(1):13-20.

53. Quante A et al. Aripiprazole as adjunct to a mood stabilizer and citalopram in bipolar depression: a randomized placebo-controlled pilot study. Hum Psychopharmacol. 2010;25(2):126-32.

54. Carlson BX et al. Aripiprazole in combination with lamotrigine for the long-term treatment of patients with bipolar I disorder (manic or mixed): a randomized, multicenter, double-blind study (CN138392). Bipolar Disord. 2012;14(1):41-53.

55. Keck Jr PE et al. A randomized, double-blind, placebo-controlled 26-week trial of aripiprazole in recently manic patients with bipolar I disorder. J Clin Psychiatry. 2006;67(4):626-37.

56. Marcus $\mathrm{R}$ et al. Efficacy of aripiprazole adjunctive to lithium or valproate in the long-term treatment of patients with bipolar I disorder with an inadequate response to lithium or valproate monotherapy: a multicenter, double-blind, randomized study. Bipolar Disord. 2011;13(2):133-44.

57. Woo YS et al. Aripiprazole plus divalproex for recently manic or mixed patients with bipolar I disorder: a 6-month, randomized, placebo-controlled, double-blind maintenance trial. Hum Psychopharmacol. 2011;26(8):543-53.

58. Lombardo I et al. Two 6-week, randomized, doubleblind, placebo-controlled studies of ziprasidone in outpatients with bipolar I depression: did baseline characteristics impact trial outcome? J Clin Psychopharmacol. 2012;32(4):470-8. 
A report of two 6-week placebo controlled RCTs (total $\mathrm{N}=885$ ) demonstrating that ziprasidone monotherapy was not effective in the treatment of acute BD-D

59. Patkar A et al. A 6 week randomized double-blind placebo-controlled trial of ziprasidone for the acute depressive mixed state. PLoS One. 2012;7(4):e34757.

60. Nierenberg AA et al. Treatment-resistant bipolar depression: a STEP-BD equipoise randomized effectiveness trial of antidepressant augmentation with lamotrigine, inositol, or risperidone. Am J Psychiatry. 2006;163(2):210-6.

61. Macfadden W et al. A randomized, double-blind, placebo-controlled study of maintenance treatment with adjunctive risperidone long-acting therapy in patients with bipolar I disorder who relapse frequently. Bipolar Disord. 2009;11(8):827-39.

62.• Loebel A et al. Lurasidone monotherapy in the treatment of bipolar I depression: a randomized, double-blind, placebo-controlled study. Am J Psychiatry. 2014;171(2):160-8.

A large 6-week placebo-controlled RCT of lurasidone (2060mg, N=166; 80-120mg, N=169; Placebo N=170) demonstrating efficacy with lurasidone monotherapy in acute BDD. Importantly, the lower dose (20-60mg) was equivalent to the higher dose. The medication was well tolerated and weight neutral

63.• Loebel A et al. Lurasidone as adjunctive therapy with lithium or valproate for the treatment of bipolar I depression: a randomized, double-blind, placebo-controlled study. Am J Psychiatry. 2014;171(2):169-77. A large $(\mathrm{N}=348)$ 6-week placebo-controlled RCT of lurasidone (20-120mg) as an adjunct to mood stabilizers demonstrating that lurasidone is efficacious in $\mathrm{BD}-\mathrm{D}$, weltolerated and weight neutral

64. Baldessarini RJ et al. Patterns of psychotropic drug prescription for U.S. patients with diagnoses of bipolar disorders. Psychiatr Serv. 2007;58(1):85-91.

65. Fu AZ et al. Second-generation antidepressant discontinuation and depressive relapse in adult patients with bipolar depression: results of a retrospective database analysis. Clin Ther. 2006;28(6):979-89.

66. Gijsman HJ et al. Antidepressants for bipolar depression: a systematic review of randomized, controlled trials. Am J Psychiatry. 2004;161(9):1537-47.

67. Sachs GS et al. Effectiveness of adjunctive antidepressant treatment for bipolar depression. N Engl J Med. 2007;356(17):1711-22.

68.• Sidor MM, Macqueen GM. Antidepressants for the acute treatment of bipolar depression: a systematic review and meta-analysis. J Clin Psychiatry. 2011;72(2):156-67.

A meta-analysis of 15 RCTs with duration 4-26 weeks $(\mathrm{N}=2,373)$ demonstrating marginal significance for response and remission with the addition of antidepressants in BD-D. SNRIs and TCAs were identified as more likely to be associated with an affective switch, whereas SSRIs and bupropion were not
69. Cipriani A et al. Comparative efficacy and acceptability of 12 new-generation antidepressants: a multiple-treatments meta-analysis. Lancet. 2009;373(9665):746-58.

70. Ghaemi SN et al. Antidepressant discontinuation in bipolar depression: a systematic treatment enhancement program for bipolar disorder (STEPBD) randomized clinical trial of long-term effectiveness and safety. J Clin Psychiatry. 2010;71(4):372-80.

71. Altshuler L et al. Impact of antidepressant discontinuation after acute bipolar depression remission on rates of depressive relapse at 1 -year follow-up. Am J Psychiatry. 2003;160(7):1252-62.

72. Post RM et al. Mood switch in bipolar depression: comparison of adjunctive venlafaxine, bupropion and sertraline. Br J Psychiatry. 2006;189:124-31.

73. Vieta E et al. A randomized trial comparing paroxetine and venlafaxine in the treatment of bipolar depressed patients taking mood stabilizers. J Clin Psychiatry. 2002;63(6):508-12.

74. Bond DJ et al. Antidepressant-associated mood elevations in bipolar II disorder compared with bipolar I disorder and major depressive disorder: a systematic review and meta-analysis. J Clin Psychiatry. 2008;69(10):1589-601.

75. Pacchiarotti I et al. The International Society for Bipolar Disorders (ISBD) task force report on antidepressant use in bipolar disorders. Am J Psychiatry. 2013;170(11):1249-62.

76. Fava M, Thase ME, DeBattista C. A multicenter, placebo-controlled study of modafinil augmentation in partial responders to selective serotonin reuptake inhibitors with persistent fatigue and sleepiness. J Clin Psychiatry. 2005;66(1):85-93.

77. Frye MA et al. A placebo-controlled evaluation of adjunctive modafinil in the treatment of bipolar depression. Am J Psychiatry. 2007;164(8):1242-9.

78. Calabrese JR et al. Adjunctive armodafinil for major depressive episodes associated with bipolar I disorder: a randomized, multicenter, double-blind, placebo-controlled, proof-of-concept study. J Clin Psychiatry. 2010;71(10):1363-70.

A well sized 8-week trial ( $\mathrm{N}=257)$ placebo controlled RCT of adjunctive armodafinil (150mg/d) demonstrating marginal improvements in depressive symptoms over placebo

79. Ketter TA. A double-blind, placebo-controlled, multicenter trial of adjunctive armodafinil for the treatment of major depression associated with bipolar I disorder. in International Conference on Bipolar Disorders. 2013. Miami Beach, FLA.

80. Goldberg JF, Burdick KE, Endick CJ. Preliminary randomized, double-blind, placebo-controlled trial of pramipexole added to mood stabilizers for treat- 
ment-resistant bipolar depression. Am J Psychiatry. 2004;161(3):564-6.

81. Zarate Jr CA et al. Pramipexole for bipolar II depression: a placebo-controlled proof of concept study. Biol Psychiatry. 2004;56(1):54-60.

82. Lesperance F et al. The efficacy of omega-3 supplementation for major depression: a randomized controlled trial. J Clin Psychiatry. 2011;72(8):1054-62.

83. Stoll AL et al. Omega 3 fatty acids in bipolar disorder: a preliminary double-blind, placebo-controlled trial. Arch Gen Psychiatry. 1999;56(5):407-12.

84. Frangou S, Lewis M, McCrone P. Efficacy of ethyleicosapentaenoic acid in bipolar depression: randomised double-blind placebo-controlled study. Br J Psychiatry. 2006;188:46-50.

85. Keck Jr PE et al. Double-blind, randomized, placebocontrolled trials of ethyl-eicosapentanoate in the treatment of bipolar depression and rapid cycling bipolar disorder. Biol Psychiatry. 2006;60(9):1020-2.

86. Murphy BL et al. Omega-3 fatty acid treatment, with or without cytidine, fails to show therapeutic properties in bipolar disorder: a double-blind, random- ized add-on clinical trial. J Clin Psychopharmacol. 2012;32(5):699-703.

87. Berk $\mathrm{M}$ et al. The efficacy of $\mathrm{N}$-acetylcysteine as an adjunctive treatment in bipolar depression: an open label trial. J Affect Disord. 2011;135(1-3):389-94.

88. Berk $\mathrm{M}$ et al. Maintenance $\mathrm{N}$-acetyl cysteine treatment for bipolar disorder: a double-blind randomized placebo controlled trial. BMC Med. 2012;10:91.

89. Diazgranados $\mathrm{N}$ et al. A randomized add-on trial of an N-methyl-D-aspartate antagonist in treatment-resistant bipolar depression. Arch Gen Psychiatry. 2010;67(8):793-802.

90. Zarate Jr CA et al. Replication of ketamine's antidepressant efficacy in bipolar depression: a randomized controlled add-on trial. Biol Psychiatry. 2012;71(11):939-46.

91. McGirr A, et al. A systematic review and meta-analysis of randomized double-blind controlled trials of ketamine in the rapid treatment of major depressive episodes. submitted. 